\title{
Changes in Gene Expression Profiling and Phenotype in Aged Multidrug Resistance Protein 4-Deficient Mouse Retina
}

\author{
Kyung Woo Kim ${ }^{1}$, Sentaro Kusuhara 1,*, Atsuko Katsuyama-Yoshikawa ${ }^{1}$, Sho Nobuyoshi ${ }^{1}$, \\ Megumi Kitamura ${ }^{1}$, Sotaro Mori ${ }^{1}$, Noriyuki Sotani ${ }^{1}$, Kaori Ueda ${ }^{1}$, Wataru Matsumiya ${ }^{1}$, Akiko \\ Miki ${ }^{1}$, Takuji Kurimoto ${ }^{1}$, Hisanori Imai ${ }^{1}$, and Makoto Nakamura ${ }^{1}$ \\ 1 Department of Surgery, Division of Ophthalmology, Kobe University Graduate School of Medicine, 7-5-1 \\ Kusunoki-cho, Chuo-ku, Kobe 650-0017, Japan; kim6048@med.kobe-u.ac.jp (K.W.K.); kusu@med.kobe- \\ u.ac.jp (S.K.); monpey_696sp@yahoo.co.jp (A.K-Y.); rainbow_milkyway_wowwow@yahoo.co.jp (S.N.); \\ megkita@med.kobe-u.ac.jp (M.K.); smori@med.kobe-u.ac.jp (S.M.); sn1117@med.kobe-u.ac.jp (N.S.); \\ kueda@med.kobe-u.ac.jp (K.U.); ytkmatsu@med.kobe-u.ac.jp (W.M.); acacyey@med.kobe-u.ac.jp (A.M.); \\ kuri1201@med.kobe-u.ac.jp (T.K.); hisimai@med.kobe-u.ac.jp (H.I.); manakamu@med.kobe-u.ac.jp (M.N.) \\ * Correspondence: kusu@med.kobe-u.ac.jp
}

\begin{abstract}
Multidrug resistance protein 4 (MRP4) is an energy-dependent membrane transporter that is responsible for cellular efflux of a broad range of xenobiotics and physiological substrates. In this trial, we aimed to investigate the co-effects of aging and MRP4 deficiency using gene expression microarray and morphological and electrophysiological analyses of the mouse retina. Mrp4knockout (null) mice and wild-type (WT) mice were reared in the same condition to 8-12 wk (young) or 45-55 wk (aged). Microarray analysis identified 186 differently expressed genes from the retinas of aged Mrp4-null mice as compared to that from aged WT mice, and subsequence gene ontology and KEGG pathway analyses showed that differently expressed genes were related to lens, eye development, vision, and transcellular barrier function that are involved in metabolic pathways or viral infection pathways. No significant change in thickness was observed for each retinal layer among young/aged WT mice and young/aged Mrp4-null mice. Moreover, immunohistochemical analyses of retinal cell type did not exhibit an overt change in the cellular morphology or distribution among the 4 age/genotype groups, and the electroretinogram responses showed no significant differences in the amplitude or the latency between aged WT mice and aged Mrp4-null mice. Aging would be an insufficient stress to cause some damage to the retina in the presence of MRP4 deficiency.
\end{abstract}

Keywords: multidrug resistance protein 4; ATP-binding cassette (ABC) transporters; aging; retina; mouse; electroretinogram.

\section{Introduction}

In general, aging affects all organ systems in individuals via several physiological and pathological processes [1]. Oxidative stress is a major modifier of organ dysfunction and has been lined to age-related diseases, such as cardiovascular diseases, diabetes, chronic kidney diseases, and neurodegenerative diseases [2-5]. A recent systematic review suggests the association of a long lifespan and less oxidative damage although the authors admitted that the complexity of the etiology and the effects of genetic-environment interaction occurring throughout the lifetime remain unclear [6]. Research has shown that oxidative stress regulates the expression of a subset of efflux ATPbinding cassette $(\mathrm{ABC})$ transporters that are located on the cell membrane that protect cells from against oxidative stress, including P-glycoprotein, multidrug resistance-related proteins, and breast cancer-related protein [7]. The retina is one of the most susceptible tissues to the oxidative damage because it has the highest oxygen consumption rate per $\mathrm{kg}$ of the body among body organs, thereby leading to exposure to a large amount of endogenous reactive oxygen species [8]. Oxidative stress 
leads to various types of ocular disorders, including retinal dystrophy [9] and mutations in $A B C A 4$, the gene coding $\mathrm{ABC}$ transporter (subfamily A, member 4), and causes retinal dystrophies, such as autosomal recessive Stargardt disease [10].

Multidrug resistance-related protein 4 (MRP4) belongs to the $C$ subfamily of $A B C$ transporters and maintains a cellular environment by functioning as an efflux pump. MRP4 transports a wide variety of endogenous and xenobiotics compounds out of cells, some of which usually induce oxidative stress $[7,11]$. Previous loss-of-function studies imply that mouse MRP4 does not play a critical physiological role because Mrp4-defficient mice reared under normal conditions are viable and do not show any overt organ dysfunction [8,12-14]. However, it is known that Mrp4-defficient mammals express abnormal reactions to various types of stress, such as carotid artery injury, hypoxia-induced pulmonary arterial hypertension, and systemic administration of forskolin, an adenylyl cyclase activator [15-17]. Thinking of the relationship between aging and oxidative stress, MRP4 may play a role in the process of aging. An experimental study wherein comprehensive mRNA profiles for drug-metabolizing enzyme and transporters were investigated showed that mRNA levels of Mrp4 in the liver were higher in aged mice [18]. In another in vivo study, MRP4 was overexpressed in the heart of aged rats, and pharmacological inhibition of MRP4 restored the positive inotropic effect of $\beta$-adrenoreceptor stimulation that was reduced in the senescent heart [19]. Moreover, in Drosophila, loss-of-function mutations of $d M R P 4$, a Drosophila homolog of human MRP4, increased the sensitivity to oxidative stress and reduced lifespan [20].

Recently, MRP4 has been recognized as an attractive pharmacological target because it contributes not only to detoxification, but also to the homeostasis of several signaling molecules. Previous xenobiotics studies identified FDA-approved drugs or bioactive compounds with inhibition potency for MRP4. FDA-approved drugs with MRP4 inhibitory function include drugs that are administered commonly in clinical practice, such as non-steroidal anti-inflammatory drugs, hypolipidemic agents, antithrombotic agents, and angiotensin converting enzyme inhibitor [11,21,22]. Therefore, aged individuals with systemic diseases might have been exposed to one of these drugs for years. The retina, used as a window to the brain, is a part of the central nervous system (CNS), and animal experiments have confirmed that MRP4 is expressed predominantly on vascular endothelial cells in the retina as in the brain $[11,23,24]$.

Although accumulating evidence on MRP4 roles in physiological and pathological conditions exist, the impact of loss of MRP4 on aging remains elusive. Therefore, to obtain insights into the coeffects of MRP4 deficiency and aging on CNS, we investigated the changes in gene expression profiles and phenotype in aged mouse retina; further, we compared these parameters between wild-type (WT) and Mrp4-null mice.

\section{Materials and Methods}

\subsection{Mice}

All the animal procedures were reviewed and approved by Kobe University Animal Care and Use Committee (permission numbers P141204 and P200406). The study was performed as per Kobe University Animal Experimentation Regulations and the ARVO Statement for the Use of Animals in Ophthalmic and Vision Research. Mrp4-knockout mice (gifted from Dr. John D. Schuetz in St. Jude Children's Research Hospital, Memphis, TN) on C57BL/6J genetic background was maintained and used. All of the mice were maintained under standard laboratory conditions $(12 / 12 \mathrm{~h}$ light/dark cycle and $20^{\circ} \mathrm{C}-24^{\circ} \mathrm{C}$; food and water were provided without restriction). For all the procedures, anesthetic effect was achieved by intraperitoneal injection of a mixture of $0.75 \mathrm{mg} / \mathrm{kg}$ medetomidine, $4.0 \mathrm{mg} / \mathrm{kg}$ midazolam, and $5.0 \mathrm{mg} / \mathrm{kg}$ butorphanol.

\subsection{Gene expression microarray}

Retinal tissues were harvested from 3 mice aged 45-55 wk and stored in an Eppendorf tube filled with TRIzol ${ }^{\circledR}$ (Thermo Fisher Scientific, Waltham, MA, USA) at $-80^{\circ} \mathrm{C}$ until RNA extraction. We prepared 4 samples for each mouse genotype (WT and $\mathrm{Mrp}^{--}{ }^{--}$). RNA extraction and the following 
RNA microarray and KEGG pathway analysis was performed by Macrogen Inc. (Tokyo, Japan). Onecolor Cy3 RNA labeling and array hybridization to Agilent SurePrint G3 Mouse Gene Expression Arrays $8 \times 60 \mathrm{~K}$ Ver. 2.0 (Agilent Technologies, Santa Clara, CA, USA) were performed. In brief, labeled cRNA was prepared from 1-5 $\mu$ g total RNA using the Agilent's Quick Amp Labeling Kit. Following fragmentation, $1.65 \mu \mathrm{g}$ of cRNA were hybridized to the Agilent expression microarray as per the protocols recommended by the manufacturer. Then, the arrays were scanned using the Agilent Technologies G4900DA SG12494263, and array data export processing and analysis was performed with Agilent Feature Extraction v11.0.1.1. The raw data for same gene was then summarized automatically in the Agilent Feature Extraction protocol to generate raw data text file, providing expression data for each gene that was probed on the array.

\subsection{Data analyses}

Array probes that have Flag A in the samples were filtered out. Selected gProcessed Signal value was transformed by logarithm and normalized using the quantile method. Statistical significance of the expression data was determined using fold change and local pooled error (LPE) test wherein the null hypothesis was that no difference exists between both the groups. Hierarchical cluster analysis was performed using complete linkage and Euclidean distance as a measure of similarity. Significant counting by the absolute value of fold change $\geq 1.5$ and P-value LPE $<0.05$ was performed using the processed data. Then, Gene-Enrichment and Functional Annotation analysis for significant probe list was performed using Gene Ontology (GO) (http://geneontology.org) and KEGG (http://kegg.jp). All data analysis and visualization of differentially expressed genes were performed using $\mathrm{R}$ 3.3.3 (www.r-project.org). Primary microarray data after significant counting have been deposited in NCBI's Gene Expression Omnibus and are accessible through GEO series accession number (GSE162376).

\subsection{Immunohistochemistry and HEE staining}

Retinal whole-amount immunostaining, immunohistochemical staining of tissue sections, and H\&E staining for frozen retinal sections were performed, as described previously [17,24,25]. Acquisition and analyses of fluorescence images were performed using a confocal laser scanning microscope (LSM-700; Carl Zeiss, Tokyo, Japan), and H\&E retinal sections were photographed with BZ-8000 (Keyence, Osaka, Japan). The primary and secondary antibodies used were rat anti-CD31 antibody (BD Pharmingen, San Diego, CA, USA), Cy3 conjugated mouse anti-glial fibrillary acidic protein (GFAP) antibody (Sigma-Aldrich), rabbit anti-glutamine synthetase antibody (Thermo Fisher Scientific, Waltham, MA, USA), rabbit anti-calbindin D-28K (Merck Millipore, Burlington, MA, USA), sheep anti-Chx10 antibody (Exalpha Biological, Shirley, MA, USA), Rhodamine-conjugated peanut agglutinin (Vector Laboratories, Burlingame, CA), Cy3 conjugated donkey anti- anti-rat IgG $(\mathrm{H}+\mathrm{L})$ antibody (Jackson ImmunoResearch, West Grove, PA, USA), Alexa Fluor 488 conjugated donkey antirat IgG antibody (Invitrogen), Alexa Fluor 488 conjugated donkey anti-rabbit IgG antibody (Invitrogen), Alexa Fluor 488 conjugated donkey anti-sheep IgG antibody (Invitrogen), and TO-PRO3 (Invitrogen). We used Image J software for quantitative analyses (AngioTool software for the analyses of vessel area and total vessel length) [26]. The thickness of each retinal layer was quantified by manually measuring the retinal layers at $100 \mu \mathrm{m}$ from the optic disc on H\&E retinal section.

\subsection{Electroretinography recording}

Electroretinography (ERG) recording were conducted, as reported previously $[27,28]$ with some modifications. All the animals were dark-adapted overnight before ERG recordings, and all the procedures were performed under dim red light. Mice were anesthetized and positioned on a built-in heating pad that maintains the body temperature at $37^{\circ} \mathrm{C}$. After dilating the pupils using $2.5 \%$ phenylephrine and 1.0\% tropicamide eye drop, (Santen Pharmaceutical Co., Osaka, Japan), a contact lens electrode embedded with gold wire was placed on the cornea as an active electrode (Mayo, Aichi, 
Japan), and a chloride silver plate was placed in the mouth as a reference electrode. The ERGs were recorded using commercially available equipment with a Ganzfeld bowl (Mayo, Aichi, Japan). Scotopic recordings were obtained from dark-adapted animals at the following increasing light intensities. Responses were amplified 10,000 times, and the band-pass filtered from 0.3 to $500 \mathrm{~Hz}$ (PuREC PC-100, Mayo). For the recording of scotopic threshold response (STR), serially increasing luminescence intensities of $-6.1,-5.5,-5.1,-4.6$, and $-4.1 \log s c t d s$ were used. Responses were amplified differentially and band-pass filtered at $0.125-50 \mathrm{~Hz}$, and responses from 80 repeated stimuli for each intensity were averaged. Photopic recordings were performed after 5-min light adaptation intervals on a background light intensity of $1.5 \mathrm{log} \mathrm{sc}$ td. Responses were amplified differentially and band-pass filtered at 0.3-300 $\mathrm{Hz}$, and the responses from 30 (20 for flicker) repeated stimuli for each intensity were averaged.

\subsection{Statistical analyses}

Statistical analyses were performed using MedCalc software version 11.3 (MedCalc Software, Mariakerke, Belgium). All the descriptive statistics are presented as mean \pm standard deviation values (all the experiments were performed at least in triplicate). Comparison between the groups and among $>3$ groups was analyzed using Student's t-test and analysis of variance (ANOVA), respectively. Student-Newman-Keuls test for all pairwise comparisons were conducted when the $\mathrm{F}$ ratio was significant in ANOVA. A P-value $<0.05$ was considered to indicate statistical significance. Microarray data analyses were conducted, as described above.

\section{Results}

\subsection{Altered gene expression profile in aged Mrp4-null mouse retina}

In order to examine comprehensive transcriptome information on aged Mrp4-null mouse retina, we performed oligonucleotide-based microarray analysis with Agilent SurePrint G3 Mouse Gene Expression Arrays 8 x 60 K Ver. 2.0 that covers the content source from RefSeq, Ensembl, RIKEN, UniGene, and GenBank databases to provide full coverage of the mouse transcriptome: 27,112 Entrez Genes, 4,578 lncRNAs, 39,430 Entrez Gene RNAs, and 16,251 lincRNAs. As compared to age-matched WT mice, 186 differently expressed genes were identified from the retinas of aged (about $50 \mathrm{wk}$ old) Mrp4-null mice with the absolute value of fold change $\geq 1.5$ and P-value of LPE $<0.05$ (Table S1). Top 10 up- or down-regulated genes have been listed in Table 1.

Table 1. Top 10 up- or down-regulated probe list in the retina of aged Mrp4 knockout mice. LPE, local pooled error; N.A., not applicable.

\begin{tabular}{|c|c|c|c|c|}
\hline \multicolumn{5}{|c|}{ Up-regulated } \\
\hline Probe ID & Gene Symbol & RefSeq Accession & Fold change & LPE \\
\hline A_52_P630867 & Abcc4 & NM_001033336 & 15.131763 & 0 \\
\hline A_51_P431785 & Myom2 & NM_008664 & 13.353321 & 0 \\
\hline A_51_P151126 & $\mathrm{Cd} 52$ & NM_013706 & 7.874139 & 0 \\
\hline A_55_P2185504 & Masp2 & NM_010767 & 4.247988 & 3.43754E-12 \\
\hline A_51_P368009 & E2f2 & NM_177733 & 3.894574 & $6.00882 \mathrm{E}-12$ \\
\hline A_51_P445153 & Spry2 & NM_011897 & 3.755293 & $5.15669 \mathrm{E}-10$ \\
\hline A_30_P01028367 & N.A. & N.A. & 3.664921 & $2.51565 \mathrm{E}-12$ \\
\hline A_51_P251402 & Tgds & NM_029578 & 3.344442 & 1.19958E-11 \\
\hline A_51_P112966 & Ch25h & NM_009890 & 3.289766 & 0.007445763 \\
\hline A_30_P01033318 & N.A. & N.A. & 3.262382 & $4.24966 \mathrm{E}-11$ \\
\hline
\end{tabular}




\begin{tabular}{|l|l|l|l|l|}
\hline \multicolumn{2}{|c|}{ Down-regulated } & \multicolumn{1}{c|}{ Fold change } & \multicolumn{1}{c|}{ LPE } \\
\hline A_55_P2003614 & Gene Symbol & \multicolumn{1}{|c|}{ RefSeq Accession } & -10.853326 & 9.48508 E-41 \\
\hline A_55_P2032445 & Slc25a37 & NM_026331 & -7.660266 & $1.35752 E-23$ \\
\hline A_51_P361286 & Agpat5 & NM_026792 & -7.499705 & 1.30354 E-29 \\
\hline A_66_P133273 & N.A. & N.A. & -6.848760 & 4.22099 E-25 \\
\hline A_52_P70381 & N.A. & N.A. & -5.933803 & $1.07479 E-21$ \\
\hline A_66_P131433 & N.A. & N.A. & -5.677915 & $3.04301 E-18$ \\
\hline A_52_P529374 & Enox1 & NM_172813 & -5.194814 & $2.29428 E-26$ \\
\hline A_30_P01031079 & N.A. & N.A. & -4.905352 & $9.37035 E-16$ \\
\hline A_52_P555603 & Apbb2 & N.A. & -4.743147 & $2.78145 E-15$ \\
\hline A_55_P2737912 & Lgi3 & NR_130157 & -4.697855 & $2.5326 E-16$ \\
\hline
\end{tabular}

Figures 1 and 2 present the clustering of the gene expression data and scatter and volume plots of the microarray analysis. GO analysis results showed that differentially expressed genes were significantly enriched in biological processes, molecular functions, and cell components: lens development in the camera-type eye, camera-type eye development, visual system development, sensory system development, eye development, sensory organ development, visual perception, sensory perception of light stimulus, lens fiber cell differentiation, lens fiber cell development, structural constituent of eye lens, structural molecule activity, uridine-diphosphatase activity, intrinsic component of autophagosome membrane, integral component of autophagosome membrane, intrinsic component of vacuolar membrane, integral component of vacuolar membrane, and integral component of synaptic vesicle membrane (Figure 2, b-d). Subsequent KEGG pathway analysis revealed that differently expressed genes were mainly involved in the "metabolic pathway," "glycerophospholipid metabolism," "herpes simplex virus 1 infection," and "Kaposi sarcoma-associated herpes virus infection" (Figure S1).

(a)

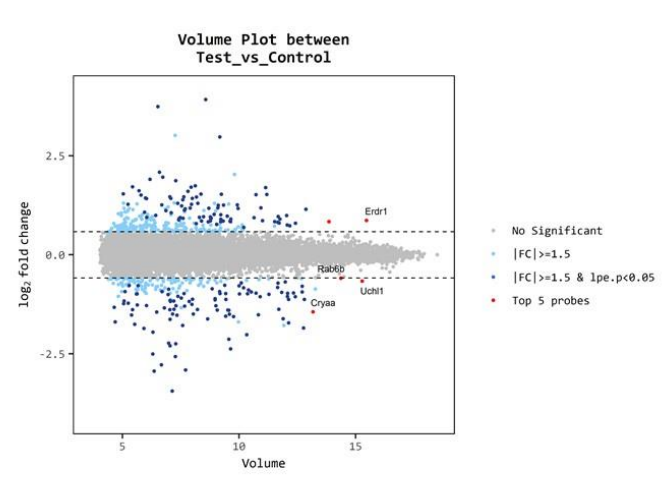

(b)

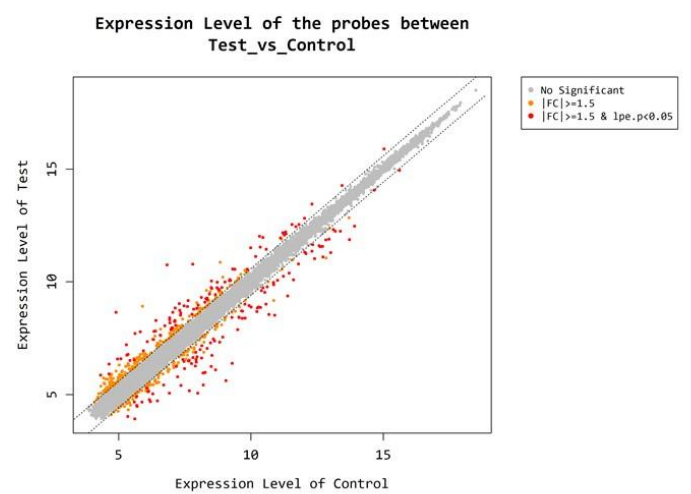

Figure 1. Volume and scatter plots of the normalized microarray data. (a) Volume plot. (b) Scatter plot. Test, Mrp4-null mice; Control, wild-type mice; fc, fold change; lpe, local pooled error. 
(a)

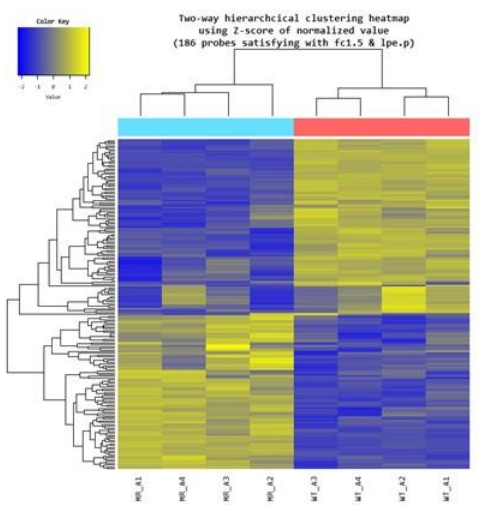

(c)

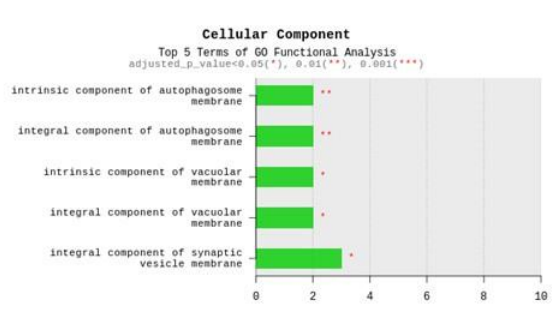

(b)

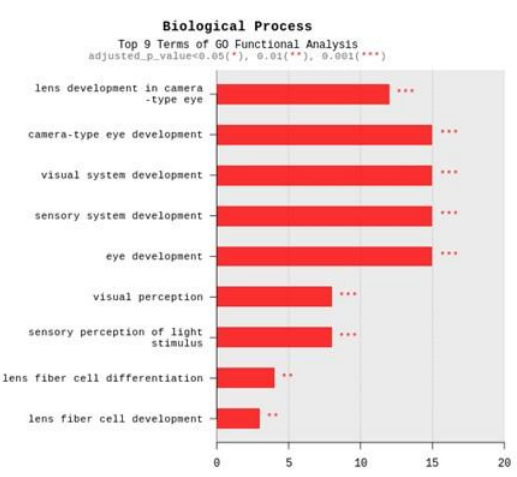

(d)

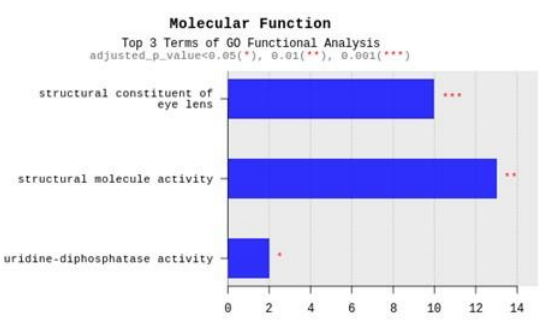

Figure 2. Microarray data analyses. (a) Cluster analysis. (b) GO analysis (biological process). (c) GO analysis (cellular component). (d) GO analysis (molecular function). Test, Mrp4-null mice; Control, wild-type mice; MR, Mrp4-null mice; WT, wild-type mice; fc, fold change; lpe, local pooled error; GO, gene ontology.

\subsection{Thickness changes in each retinal layer}

We hypothesized that differently expressed genes in aged Mrp4-null mouse retina have some associations with the retinal architecture. Therefore, we then assessed the thickness changes in each retinal layer: nerve fiber layer (NFL)/ ganglion cell layer (GCL) complex, inner plexiform layer (IPL), inner nuclear layer (INL), outer plexiform layer (OPL), outer nuclear layer (ONL), and photoreceptor layer (PhR). Figure 3 shows the results of H\&E staining. No significant change in the thickness was observed for each retinal layer among young (8-12 wk) WT mice, aged (45-55 wk) WT mice, young Mrp4-null mice, and aged Mrp4-null mice.

\section{(a)}

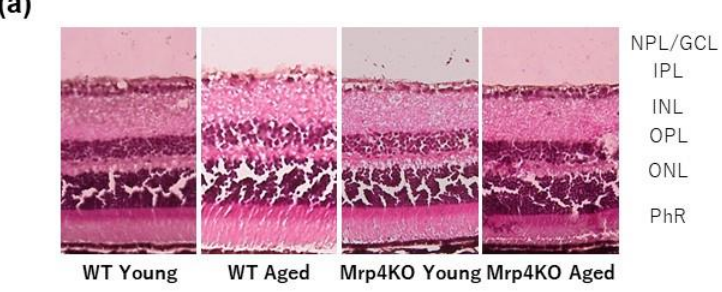

(b)

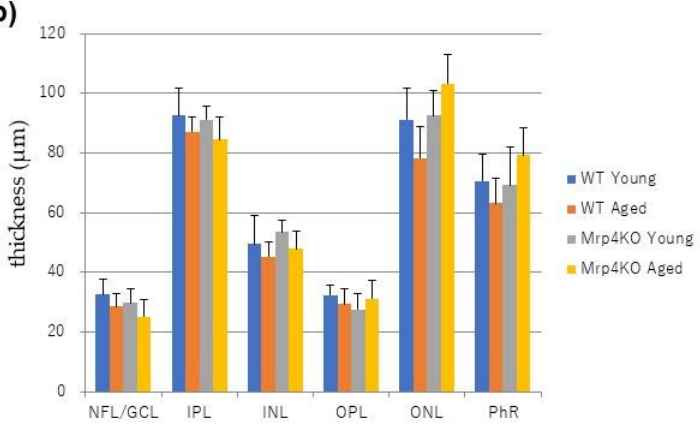

Figure 3. H\&E staining results. (a) Representative retinal section images of H\&E staining. (b) Quantitative comparison of thickness in each retinal layer. No significant difference was observed $(\mathrm{n}=$ 
6). WT, wild-type mice; Mrp4KO, Mrp4-null mice; NFL, nerve fiber layer (NFL); GCL, ganglion cell layer; IPL, inner plexiform layer; INL, inner nuclear layer; OPL, outer plexiform layer; ONL, outer nuclear layer; PhR, photoreceptor layer.

\subsection{Morphology and distribution of each retinal cell type}

In response to the results of H\&E staining, we checked the morphology and distribution of each retinal cell type because the changes related to aging and Mrp4 deficiency might be detected at the cellular level. Section immunohistochemical staining demonstrated no apparent changes in the morphology and distribution among young/aged WT mice and young/aged Mrp4-null mice for vascular endothelial cells (CD31), astrocytes (GFAP), Muller cells (glutamine synthetase), horizontal and amacrine cells (Calbindin), bipolar cells (Chx10), or photoreceptors (peanut agglutinin) (Figure 4).



Figure 4. Immunohistochemical staining results of the retinal section. Bar $=100 \mu \mathrm{m}$. WT, wild-type mice; Mrp4KO, Mrp4-null mice; GFAP, glial fibrillary acidic protein; GS, glutamine synthetase; PNA, peanut agglutinin.

Retinal whole mount immunostaining was performed for the two-dimensional evaluation of retinal vessels and astrocyte network that could not be used for performing section immunohistochemistry. Further, the results showed no overt change in young/aged WT mice and young/aged Mrp4-null mice in terms of the retinal vasculature or astrocyte network although some layers showed a small but significant difference in the vascular area and/or total vascular length (Figures 5 and 6). 


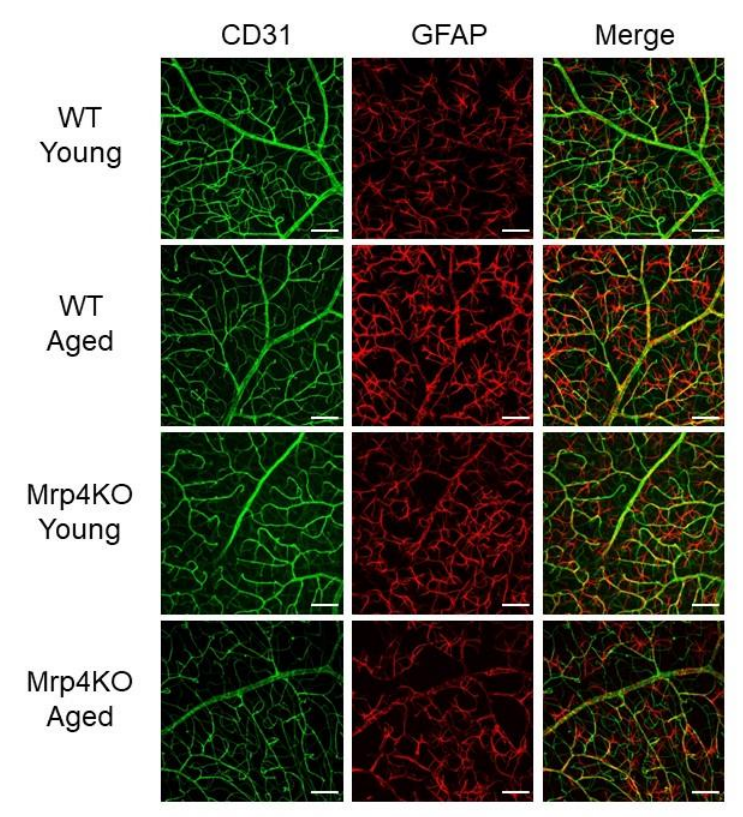

Figure 5. Immunohistochemical staining results of whole mount retina. Retinal vasculature and astrocyte network at the superficial layer of the retina. Bar $=100 \mu \mathrm{m}$.
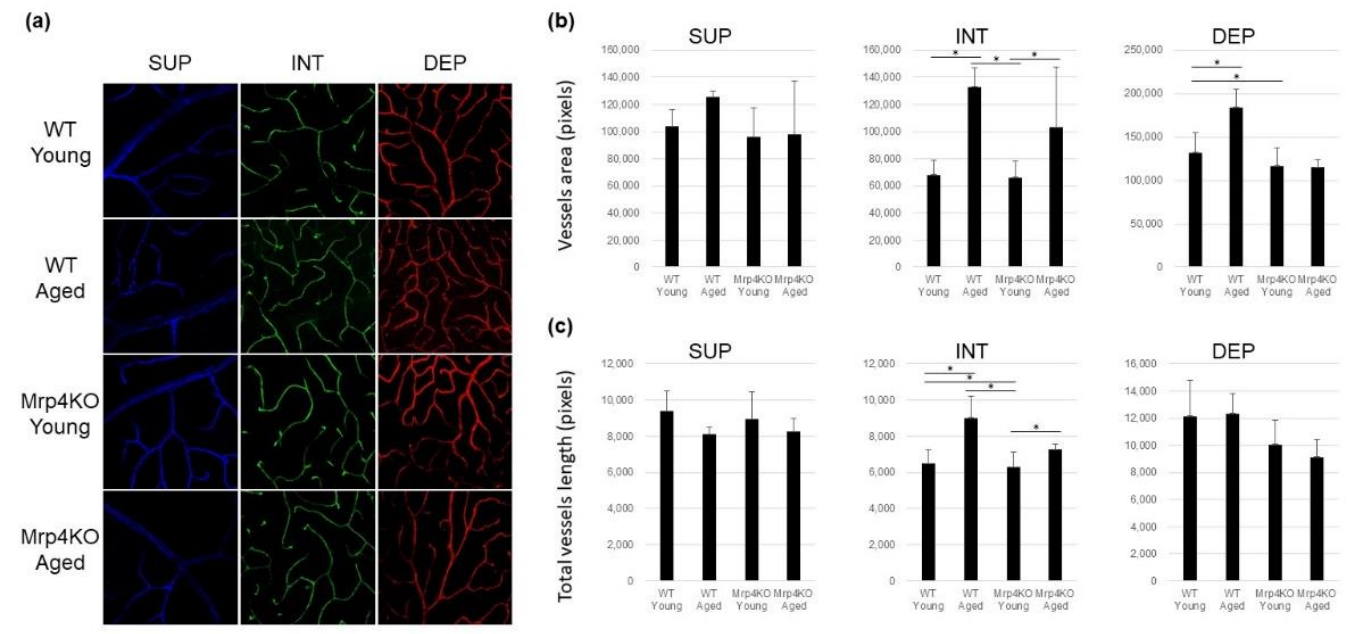

Figure 6. Retinal vasculature at the different levels of the retinal layer. (a) Representative images (x20 objective). (b) Quantitative comparison of vessel area in each retinal layer $(n=3-4)$. (c) Quantitative comparison of the total vessel length in each retinal layer ( $n=3-4)$. WT, wild-type mice; Mrp4KO, Mrp4null mice; GFAP, glial fibrillary acidic protein; SUP, superficial retinal layer; INT, intermediate retinal layer; DEP, deep retinal layer. ${ }^{*} \mathrm{P}<0.05$.

\subsection{Electrophysiological function of the retina}

In order to test whether the retina of aged Mrp4-null mice has the same retinal function as that of WT mice, we conducted electrophysiological analyses. Electroretinography (ERG) performed for aged Mrp4-null mice showed no significant difference from aged WT mice in terms of the amplitude and latency of a-wave, b-wave, and the amplitude of positive STR (Figure 7 and Figure S2). 
(a)

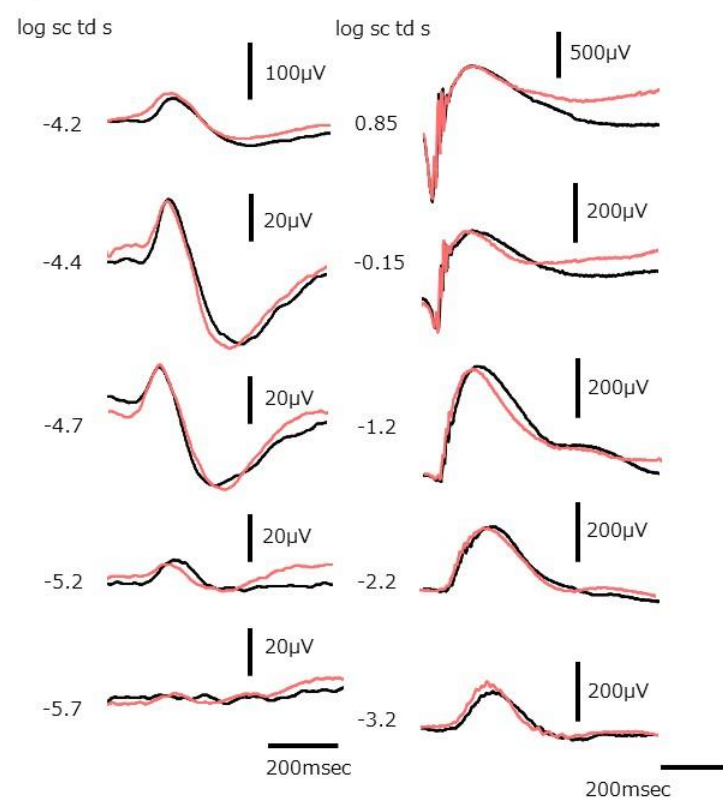

(b)

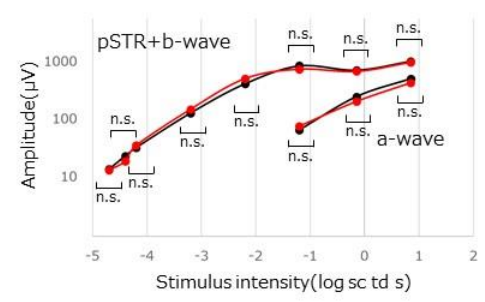

(c)

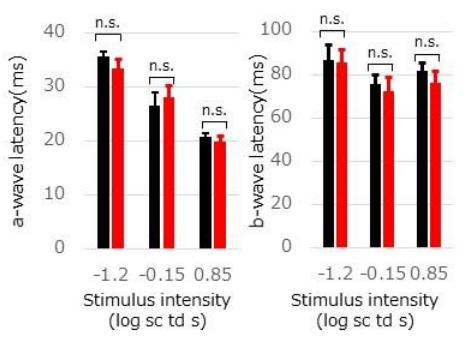

Figure 7. Results of scotopic electroretinography (ERG) phenotype. (a) Representative dark-adapted ERG recordings. (b) Quantitative analyses of the amplitude of PSTR + b-wave $(n=6)$. (c) Quantitative analyses of the latency of $a-$ and $b$-waves $(n=6)$. Error bar $=$ standard deviation. Black line or black bars represent the data from aged ( $45 \mathrm{wk}$ old) WT mice and red line or red bars represent the data from aged (45 wk old) Mrp4-null mice. pSTR, positive scotopic threshold response; n.s., not significant.

\section{Discussion}

In the current study, we performed a comprehensive transcriptome analysis and identified 186 differently expressed genes in aged Mrp4-null mice as compared to that in aged WT mice and obtained the information on significant GO terms and KEGG pathways using the differently expressed gene. However, no overt retinal phenotype related to aging or Mrp4 deficiency was observed in the retinal architecture, morphology, and distribution of each retinal cell type or electrophysiological function.

A total of 186 differently expressed genes would reflect the effects of the blood-retinal barrier (BRB) dysfunction on the neural retina because (1) MRP4 is almost exclusively expressed in vascular endothelial cells in the retina [23,24], (2) endothelial cells are major components of the BRB [29,30], (2) MRP4 restricts transcellular transport [22], and (4) endothelial cells account for only a small cell population $(0.1 \%)$ in the retina [31]. The GO terms significantly classified using 186 differently expressed genes involved those related to the lens (lens development in camera-type eye, lens fiber cell differentiation, and lens fiber cell development), eye development (camera-type eye development, visual system development, sensory system development, eye development, and sensory organ development), and vision (visual perception and sensory perception of light stimulus) for biological process. We confirmed that the extracted GO terms in our study varied from those in other studies that investigated the effect of ocular inflammation, ultraviolet radiation, or strain [32-34]. It surprised us that differently expressed genes were enriched within lens-related GO because the retina and lens are being recognized as two entirely different tissue types. However, this result might convey an important message to us. For example, crystallins are primarily found as major structural proteins of the ocular lens; however, it was then disclosed that $\alpha \mathrm{B}$-crystallin acts as a regulator of retinal angiogenesis [35]. The significant GO terms for molecular function or cellular components, such as structural molecule activity, intrinsic component of vacuolar membrane, and integral component of synaptic vesicle membrane might be associated to the MRP4 transcellular barrier function in the CNS [36]. Specific signaling pathway affected by Mrp4 deficiency in aged mouse retina included "metabolic pathway," "glycerophospholipid metabolism," "herpes simplex virus 1 infection," and "Kaposi sarcomaassociated herpes virus infection." Although distinct relationships between the former 2 pathways and 
MRP4 remain unknown, the latter 2 pathways might be associated with MRP4 function as an efflux transporter because antiviral agents are MRP4 substrates [22,37].

Before focusing on some key genes that were significantly up-regulated or down-regulated in aged Mrp4-null mice in the list, we checked the severity of the impact on the retina aging and Mrp4 deficiency. Thickness change in the specific retinal layer is likely to be a good marker of retinal degeneration [38]; therefore, we measured the thickness of retinal layers in young/aged WT mice and young/aged Mrp4null mice and performed a comparison. Unexpectedly, although $>100$ of the genes are differently expressed in aged Mrp4-null mouse retinas, changes in the thickness of each retinal layer did not show any significant differences. We checked some retinal sections from WT and Mrp4-null mice aged $2 \mathrm{y}$ and found no overt retinal phenotype (data not shown). Moreover, immunohistochemical analyses of the retinal cell type did not exhibit any obvious change in the cellular morphology or distribution among the 4 age/genotype mouse groups. Inconspicuous vascular phenotypes observed in the intermediate and/or deep retinal layer might be attributed to Mrp4 deficiency, aging, or both because we previously disclosed that Mrp4 deficiency led to retinal vascular phenotypes after forskolin administration in neonatal mice [17]. An extensive electrophysiological analysis confirmed these histological results, showing no significant ERG responses in the amplitude or the latency between the aged WT mice and aged Mrp4-null mice. Based on these experimental results, we concluded that aging is an insufficient stress to cause some damage to the retina in Mrp4-null mice. However, we are currently unable to explain the reasons why aged Mrp4-null mice show no evident retinal phenotype in spite of the presence of many differently expressed genes in the retina. We made the following speculations: (1) as the mice had been housed under well-controlled and specific-pathogen-free condition, accumulated endogenous and exogenous stresses were beneath the threshold that caused retinal damage, (2) other efflux transporter family proteins expressed in the retina compensated for the lack of MRP4, and (3) the pleiotropic effects of the loss of MRP4 function canceled the molecular pathways causing retinal dysfunction. In any case, the point is that co-effects of MRP4 deficiency and aging unlikely exert serious damage to the retina. It might be a boon to patients who continue taking drugs with MRP4 inhibitory activity; however, further studies should be performed to find a resolution of this issue because they have systemic diseases, such as hyperlipidemia, hypertension, and cancer, that may impose another stress in addition to aging [11,21,22].

This study has certain limitations. First, microarray analysis was performed at only one time point. If microarray data was acquired at many different time points, the co-effects of MRP4 and chronological aging on retinal phenotype could be further understood. Second, we used whole retinas to investigate the differences in gene expression. Although the major component of the retina is the neuron, the retina has several types of neurons as well as glial cells and vascular endothelial cells. Therefore, we observed the sum of gene expressions derived from different cell types in our microarray data. Third, although technically difficult, we did not evaluate cell behaviors that would facilitate brain researchers because the retina is an ideal tissue for in vivo imaging, and the cellular events that occurred in the retina were linked to those in the brain.

In conclusion, MRP4 deficiency in aged mice caused different gene expression than in WT mice. The analyzed gene profiles suggest that aging and MRP4 deficiency affects the gene expressions in the retina associated with the lens, eye development, vision, and transcellular barrier function that are involved in metabolic pathways or viral infection pathways. However, the magnitude of the impact of gene expression changes observed in aged Mrp4-null mice on the retinal morphology and function would be small. Aging is insufficient as a stress to cause some damage to the retina in Mrp4-null mice, and further studies should be performed to investigate the co-effects of Mrp4 deficiency and other types of stress, such as hyperglycemia and inflammation.

Supplementary Materials: The following are available online at www.mdpi.com/xxx/s1, Figure S1: KEGG pathway analysis result, Figure S2: Results of photopic electroretinogram (ERG) phenotype, Table S1: Differentially expressed gene list.

Author Contributions: Conceptualization, S.K.; methodology, S.K., S.M.; formal analysis, S.K., S.M., K.W.K; investigation, K.W.K., A.K-Y, S.N., M.K., N.S.; writing-original draft preparation, S.K.; writing-review and editing, W.M., K.U., T.K., M.N.; visualization, K.W.K, S.K.; supervision, W.M., K.U., T.K., H.I., M.N.; project 
administration, S.K., M.N.; funding acquisition, S.K. All authors have read and agreed to the published version of the manuscript.

Funding: This work was supported by the Japan Society for the Promotion of Science (grant number [Grants-inAid for Young Scientists (B) 24791854 and Scientific Research (C) 15K10865 and 18K09409] to S. Kusuhara).

Acknowledgments: RNA extraction and the following RNA microarray and KEGG pathway analysis was provided by Macrogen Inc. (Tokyo, Japan).

Conflicts of Interest: The authors declare no conflict of interest.

\section{References}

1. da Costa, J.P.; Vitorino, R.; Silva, G.M.; Vogel, C.; Duarte, A.C.; Rocha-Santos, T. A synopsis on agingTheories, mechanisms and future prospects. Ageing Res Rev 2016, 29, 90-112, doi:10.1016/j.arr.2016.06.005.

2. $\quad$ El Assar, M.; Angulo, J.; Rodriguez-Manas, L. Oxidative stress and vascular inflammation in aging. Free Radic Biol Med 2013, 65, 380-401, doi:10.1016/j.freeradbiomed.2013.07.003.

3. Schrag, M.; Mueller, C.; Zabel, M.; Crofton, A.; Kirsch, W.M.; Ghribi, O.; Squitti, R.; Perry, G. Oxidative stress in blood in Alzheimer's disease and mild cognitive impairment: a meta-analysis. Neurobiol Dis 2013, 59, 100-110, doi:10.1016/j.nbd.2013.07.005.

4. Jha, J.C.; Banal, C.; Chow, B.S.; Cooper, M.E.; Jandeleit-Dahm, K. Diabetes and Kidney Disease: Role of Oxidative Stress. Antioxid Redox Signal 2016, 25, 657-684, doi:10.1089/ars.2016.6664.

5. Gerber, P.A.; Rutter, G.A. The Role of Oxidative Stress and Hypoxia in Pancreatic Beta-Cell Dysfunction in Diabetes Mellitus. Antioxid Redox Signal 2017, 26, 501-518, doi:10.1089/ars.2016.6755.

6. Belenguer-Varea, A.; Tarazona-Santabalbina, F.J.; Avellana-Zaragoza, J.A.; Martinez-Reig, M.; MasBargues, C.; Ingles, M. Oxidative stress and exceptional human longevity: Systematic review. Free Radic Biol Med 2020, 149, 51-63, doi:10.1016/j.freeradbiomed.2019.09.019.

7. Grewal, G.K.; Kukal, S.; Kanojia, N.; Saso, L.; Kukreti, S.; Kukreti, R. Effect of Oxidative Stress on ABC Transporters: Contribution to Epilepsy Pharmacoresistance. Molecules 2017, 22, doi:10.3390/molecules22030365.

8. Leggas, M.; Adachi, M.; Scheffer, G.L.; Sun, D.; Wielinga, P.; Du, G.; Mercer, K.E.; Zhuang, Y.; Panetta, J.C.; Johnston, B., et al. Mrp4 confers resistance to topotecan and protects the brain from chemotherapy. Mol Cell Biol 2004, 24, 7612-7621, doi:10.1128/MCB.24.17.7612-7621.2004.

9. E, B.D.; Marfany, G. The Relevance of Oxidative Stress in the Pathogenesis and Therapy of Retinal Dystrophies. Antioxidants (Basel) 2020, 9, doi:10.3390/antiox9040347.

10. Cremers, F.P.M.; Lee, W.; Collin, R.W.J.; Allikmets, R. Clinical spectrum, genetic complexity and therapeutic approaches for retinal disease caused by ABCA4 mutations. Prog Retin Eye Res 2020, 79, 100861, doi:10.1016/j.preteyeres.2020.100861.

11. Russel, F.G.; Koenderink, J.B.; Masereeuw, R. Multidrug resistance protein 4 (MRP4/ABCC4): a versatile efflux transporter for drugs and signalling molecules. Trends Pharmacol Sci 2008, 29, 200-207, doi:10.1016/j.tips.2008.01.006.

12. Mennone, A.; Soroka, C.J.; Cai, S.Y.; Harry, K.; Adachi, M.; Hagey, L.; Schuetz, J.D.; Boyer, J.L. Mrp4-/mice have an impaired cytoprotective response in obstructive cholestasis. Hepatology 2006, 43, 10131021, doi:10.1002/hep.21158.

13. Belinsky, M.G.; Guo, P.; Lee, K.; Zhou, F.; Kotova, E.; Grinberg, A.; Westphal, H.; Shchaveleva, I.; KleinSzanto, A.; Gallo, J.M., et al. Multidrug resistance protein 4 protects bone marrow, thymus, spleen, and 
intestine from nucleotide analogue-induced damage. Cancer Res 2007, 67, 262-268, doi:10.1158/00085472.CAN-06-2680.

14. Lin, Z.P.; Zhu, Y.L.; Johnson, D.R.; Rice, K.P.; Nottoli, T.; Hains, B.C.; McGrath, J.; Waxman, S.G.; Sartorelli, A.C. Disruption of cAMP and prostaglandin E2 transport by multidrug resistance protein 4 deficiency alters cAMP-mediated signaling and nociceptive response. Mol Pharmacol 2008, 73, 243-251, doi:10.1124/mol.107.039594.

15. Sassi, Y.; Lipskaia, L.; Vandecasteele, G.; Nikolaev, V.O.; Hatem, S.N.; Cohen Aubart, F.; Russel, F.G.; Mougenot, N.; Vrignaud, C.; Lechat, P., et al. Multidrug resistance-associated protein 4 regulates cAMP-dependent signaling pathways and controls human and rat SMC proliferation. J Clin Invest 2008, 118, 2747-2757, doi:10.1172/JCI35067.

16. Hara, Y.; Sassi, Y.; Guibert, C.; Gambaryan, N.; Dorfmuller, P.; Eddahibi, S.; Lompre, A.M.; Humbert, M.; Hulot, J.S. Inhibition of MRP4 prevents and reverses pulmonary hypertension in mice. J Clin Invest 2011, 121, 2888-2897, doi:10.1172/JCI45023.

17. Matsumiya, W.; Kusuhara, S.; Hayashibe, K.; Maruyama, K.; Kusuhara, H.; Tagami, M.; Schuetz, J.D.; Negi, A. Forskolin modifies retinal vascular development in Mrp4-knockout mice. Invest Ophthalmol Vis Sci 2012, 53, 8029-8035, doi:10.1167/iovs.12-10781.

18. Fu, Z.D.; Csanaky, I.L.; Klaassen, C.D. Effects of aging on mRNA profiles for drug-metabolizing enzymes and transporters in livers of male and female mice. Drug Metab Dispos 2012, 40, 1216-1225, doi:10.1124/dmd.111.044461.

19. Carillion, A.; Feldman, S.; Jiang, C.; Atassi, F.; Na, N.; Mougenot, N.; Besse, S.; Hulot, J.S.; Riou, B.; Amour, J. Overexpression of cyclic adenosine monophosphate effluent protein MRP4 induces an altered response to beta-adrenergic stimulation in the senescent rat heart. Anesthesiology 2015, 122, 334342, doi:10.1097/ALN.0000000000000526.

20. Huang, H.; Lu-Bo, Y.; Haddad, G.G. A Drosophila ABC transporter regulates lifespan. PLoS Genet 2014, 10, e1004844, doi:10.1371/journal.pgen.1004844.

21. Cheung, L.; Yu, D.M.; Neiron, Z.; Failes, T.W.; Arndt, G.M.; Fletcher, J.I. Identification of new MRP4 inhibitors from a library of FDA approved drugs using a high-throughput bioluminescence screen. Biochem Pharmacol 2015, 93, 380-388, doi:10.1016/j.bcp.2014.11.006.

22. Berthier, J.; Arnion, H.; Saint-Marcoux, F.; Picard, N. Multidrug resistance-associated protein 4 in pharmacology: Overview of its contribution to pharmacokinetics, pharmacodynamics and pharmacogenetics. Life Sci 2019, 231, 116540, doi:10.1016/j.lfs.2019.06.015.

23. Tachikawa, M.; Toki, H.; Tomi, M.; Hosoya, K. Gene expression profiles of ATP-binding cassette transporter A and C subfamilies in mouse retinal vascular endothelial cells. Microvasc Res 2008, 75, 6872, doi:10.1016/j.mvr.2007.05.002.

24. Tagami, M.; Kusuhara, S.; Honda, S.; Tsukahara, Y.; Negi, A. Expression of ATP-binding cassette transporters at the inner blood-retinal barrier in a neonatal mouse model of oxygen-induced retinopathy. Brain Res 2009, 1283, 186-193, doi:10.1016/j.brainres.2009.05.095.

25. Katsuyama, A.; Kusuhara, S.; Asahara, S.I.; Nakai, S.I.; Mori, S.; Matsumiya, W.; Miki, A.; Kurimoto, T.; Imai, H.; Kido, Y., et al. En face slab optical coherence tomography imaging successfully monitors progressive degenerative changes in the innermost layer of the diabetic retina. BMJ Open Diabetes Res Care 2020, 8, doi:10.1136/bmjdrc-2019-001120.

26. Zudaire, E.; Gambardella, L.; Kurcz, C.; Vermeren, S. A computational tool for quantitative analysis of vascular networks. PLoS One 2011, 6, e27385, doi:10.1371/journal.pone.0027385. 
27. Moshiri, A.; Gonzalez, E.; Tagawa, K.; Maeda, H.; Wang, M.; Frishman, L.J.; Wang, S.W. Near complete loss of retinal ganglion cells in the math $5 /$ brn3b double knockout elicits severe reductions of other cell types during retinal development. Dev Biol 2008, 316, 214-227, doi:10.1016/j.ydbio.2008.01.015.

28. Mori, S.; Kurimoto, T.; Miki, A.; Maeda, H.; Kusuhara, S.; Nakamura, M. Aqp9 Gene Deletion Enhances Retinal Ganglion Cell (RGC) Death and Dysfunction Induced by Optic Nerve Crush: Evidence that Aquaporin 9 Acts as an Astrocyte-to-Neuron Lactate Shuttle in Concert with Monocarboxylate Transporters To Support RGC Function and Survival. Mol Neurobiol 2020, 57, 4530-4548, doi:10.1007/s12035-020-02030-0.

29. Ogura, S.; Kurata, K.; Hattori, Y.; Takase, H.; Ishiguro-Oonuma, T.; Hwang, Y.; Ahn, S.; Park, I.; Ikeda, W.; Kusuhara, S., et al. Sustained inflammation after pericyte depletion induces irreversible bloodretina barrier breakdown. JCI Insight 2017, 2, e90905, doi:10.1172/jci.insight.90905.

30. Kusuhara, S.; Fukushima, Y.; Ogura, S.; Inoue, N.; Uemura, A. Pathophysiology of Diabetic Retinopathy: The Old and the New. Diabetes Metab J 2018, 42, 364-376, doi:10.4093/dmj.2018.0182.

31. Kusuhara, S.; Fukushima, Y.; Fukuhara, S.; Jakt, L.M.; Okada, M.; Shimizu, Y.; Hata, M.; Nishida, K.; Negi, A.; Hirashima, M., et al. Arhgef15 promotes retinal angiogenesis by mediating VEGF-induced Cdc42 activation and potentiating RhoJ inactivation in endothelial cells. PLoS One 2012, 7, e45858, doi:10.1371/journal.pone.0045858.

32. Qiu, Y.; Yu, P.; Lin, R.; Fu, X.; Hao, B.; Lei, B. Genome-wide retinal transcriptome analysis of endotoxininduced uveitis in mice with next-generation sequencing. Mol Vis 2017, 23, 395-406.

33. An, M.J.; Kim, C.H.; Nam, G.Y.; Kim, D.H.; Rhee, S.; Cho, S.J.; Kim, J.W. Transcriptome analysis for UVB-induced phototoxicity in mouse retina. Environ Toxicol 2018, 33, 52-62, doi:10.1002/tox.22494.

34. Wang, J.; Geisert, E.E.; Struebing, F.L. RNA sequencing profiling of the retina in C57BL/6J and DBA/2J mice: Enhancing the retinal microarray data sets from GeneNetwork. Mol Vis 2019, 25, 345-358.

35. Kase, S.; He, S.; Sonoda, S.; Kitamura, M.; Spee, C.; Wawrousek, E.; Ryan, S.J.; Kannan, R.; Hinton, D.R. alphaB-crystallin regulation of angiogenesis by modulation of VEGF. Blood 2010, 115, 3398-3406, doi:10.1182/blood-2009-01-197095.

36. Zhang, Y.; Schuetz, J.D.; Elmquist, W.F.; Miller, D.W. Plasma membrane localization of multidrug resistance-associated protein homologs in brain capillary endothelial cells. J Pharmacol Exp Ther 2004, 311, 449-455, doi:10.1124/jpet.104.068528.

37. Liu, Y.T.; Liu, W.; Zhu, G.Y.; Wang, F.L.; Chen, Q. Involvement of multidrug resistance protein 4 in the hepatocyte efflux of lamivudine and entecavir. Mol Med Rep 2018, 17, 7113-7121, doi:10.3892/mmr.2018.8779.

38. Nishiguchi, K.M.; Carvalho, L.S.; Rizzi, M.; Powell, K.; Holthaus, S.M.; Azam, S.A.; Duran, Y.; Ribeiro, J.; Luhmann, U.F.; Bainbridge, J.W., et al. Gene therapy restores vision in rd1 mice after removal of a confounding mutation in Gpr179. Nat Commun 2015, 6, 6006, doi:10.1038/ncomms7006. 